\title{
Analytic perturbative theories in highly inhomogeneous gravitational fields
}

\author{
Marta Ceccaroni \\ marta.ceccaroni@strath.ac.uk \\ Advanced Space Concepts Laboratory \\ University of Strathclyde, Glasgow \\ James Biggs \\ james.biggs@strath.ac.uk \\ Advanced Space Concepts Laboratory \\ University of Strathclyde, Glasgow
}

\section{Abstract}

Orbital motion about irregular bodies is highly nonlinear due to inhomogeneities in the gravitational field. Classical theories of motion close to spheroidal bodies cannot be applied as for inhomogeneous bodies the Keplerian forces do not provide a good approximation of the system dynamics. In this paper a closed form, analytical method for developing the motion of a spacecraft around small bodies is presented, for the so called fast rotating case, which generalize previous results to second order, arbitrary degree, gravitational fields. Through the application of two different Lie transformations, suitable changes of coordinates are found, which reduce the initial non integrable Hamiltonian of the system into an integrable one plus a negligible, perturbative remainder of higher degree. In addition, an explicit analytical formulation for the relegated, first and second order, arbitrary degree Hamiltonian for relatively high altitude motion in any inhomogeneous gravi- 
tational field is derived in closed-form. Applications of this algorithm include a method for determining initial conditions for frozen orbits around any irregular body by simply prescribing the desired inclination and eccentricity of the orbit. This method essentially reduces the problem of computing frozen orbits to a problem of solving a 2-D algebraic equation. Results are shown for the asteroid 433-Eros.

Keywords: Asteroids, dynamics, Orbit determination, Asteroids, Celestial mechanics, Irregular satellites

\section{Introduction}

The motion of bodies subject to non-Keplerian gravitational fields is a classical subject of research in the context of celestial mechanics. In recent years this type of research has become important to future planned missions of spacecraft to the moon and asteroids in addition to asteroid deflection missions such as the European Space Agency's "Don Quijote" concept Carnelli and Gálvez (2006). Research undertaken in this area has studied the effect of the Earth's inhomogeneous gravitational field on the motion of natural and artificial satellites, that is, artificial satellite theory for small and moderate eccentricities Deprit (1970). More recent studies have researched the effects on motion of the inhomogeneous gravitational field of other solar system bodies, including the Moon Abad et al. (2009) and asteroids San-Juan et al. (2004). The analysis of spacecraft motion about these bodies is particularly challenging as they typically feature shapes and density distributions more irregular than those of planets. Such irregularities break symmetries and require more complicated analytical expressions for their description which 
increases the complexity involved in such studies.

Numerical methods are today widely used to study the trajectories of objects orbiting specific irregular bodies Fahnestock and Scheeres (2008) or for finding stability criteria (Lara and Scheeres (2002)). Disadvantages of these methods are that they can be highly computational and require a complete re-design for each different body. Analytical methods, by contrast, have the potential to rapidly identify useful natural motions for general bodies with inhomogeneous gravitational fields. Furthermore, they can provide a full dynamical picture of the motion around irregular bodies that can be used to search and study particular classes of useful orbits. However, current analytical methods are only used in a limited and semi-numerical way (meaning that analytical expansions constitute the first step in such studies, which are then typically carried out from a numerical standpoint Scheeres et al. (1998)). The main drawbacks of these methods is that their application in the case of highly inhomogeneous bodies requires extensive symbolic computations involving algebraic manipulations, and that they are usually restricted to a certain range of eccentricities due to series convergence. Analytical studies on inhomogeneous gravitational fields have been, so far, limited to low degree gravity fields Palacián (2002), San-Juan et al. (2002), San-Juan et al. (2004), thus restricting the results to a class of bodies for which the dynamics is dominated by a few coefficients (e.g. oblateness or ellipticity).

In this paper a closed form (i.e. without using series expansion in the eccentricity), analytical, perturbative theory of motion around inhomogeneous bodies is presented, generalized to second order, arbitrary degree gravity fields. 
52

Using Deprit and Palacián's relegation algorithm (Palacián (1992)) and a Delaunay normalization, suitable canonical action-angle variables are found, which reduce the initial non-integrable Hamiltonian into an integrable one plus a negligible, perturbative remainder.

The method can be used to find useful orbits for space mission applications such as frozen orbits. Moreover, frozen orbits are orbits with no secular perturbations in the inclination, argument of pericenter, and eccentricity (Brouwer (1959)). These orbits are periodic orbits, except for the orbital plane of precession, and are therefore called frozen. In particular, this paper extends previous work by:

- Formulating the inhomogeneous gravitational potential generated by any inhomogeneous body in polar-nodal coordinates

- Including arbitrary degree gravitational coefficients, instead of limiting the study to $2^{\text {nd }}$ degree coefficients

- Stating the explicit analytical formulation for the closed-form averaged with respect to the argument of node, second order, arbitrary degree Hamiltonian of any inhomogeneous gravitational field.

- Obtaining a resulting Hamiltonian which accounts for the presence of the angular momentum, in contrast to the trivially integrable Hamiltonian of San-Juan et al. (2002) which only accounts for the argument of node. Again, this previous result was only possible by considering a Hamiltonian with 2nd degree coefficients. 
- Providing a method for determining initial conditions for frozen orbits around any irregular body by simply prescribing the inclination and eccentricity of the desired orbit.

- Applying the method to the asteroids 433-Eros, which is the main example studied in.

Therefore, the proposed perturbative theory presents a method to derive more accurate descriptions of a spacecraft's high altitude motion about an asteroid, which enables, for example, one to find precise initial conditions that yield frozen orbits.

\section{Method}

Assuming that the planetary body is in uniform rotation around its axis of greatest inertia the potential generated by the inhomogeneous gravitational field can be derived in the rotating polar nodal variables (Whittaker (1917)) convenient for the successive transformation to Delaunay coordinates. This potential takes into account an arbitrary number of spherical harmonic coefficients, all considered to have the same order, thus providing a dynamical model based on an arbitrarily accurate model of the inhomogeneous body. Restricting the analysis to the fast rotation case, i.e. when the angular velocity of rotation the asteroid is higher than the mean motion of the spacecraft, the methodology is then based on the following steps:

- Relegation of the polar component of the angular momentum $N$ to obtain the relegated nodal variables where the argument of nodes conjugate momenta is constant along the Hamiltonian flow. 
- Transformation to Delaunay variables to yield a constant total angular momentum in the z-direction

- Normalization of the Delaunay variables which yields a reduced ordinary differential equation in two coordinates; the total angular momentum and the argument of pericentre

- The frozen orbits are identified with the equilibrium points of these equations i.e. where the total angular momentum about the z-axis and the argument of pericentre are constant, therefore the final stage is undertaken by solving a 2-D algebraic equation.

The methodology comprises of two different Lie transformations, relegation and normalisation, constructed following Deprit and Palacián's algorithm (Palacián (1992)) and Deprit's method for Lie transformations (Deprit (1969)). The Delaunay normalization (Deprit (1982)), cannot be directly applied to a high-order model due to the presence of the argument of node that appears in the Coriolis term. The addition of this term in the Lie derivative prevents the conventional computation of the Lie transform generator (SanJuan et al. (2002)). However, Deprit and Palacián's closed form relegation algorithm (Deprit et al. (2001)) can be applied, which "relegates" the action of the argument of node to a negligible remainder. It is shown that, for this model, both relegation and normalization results are equivalent to averaging over the fast angles. 


\section{The dynamical system}

An inhomogeneous body is considered, which rotates uniformly around its axes of greatest inertia with constant angular velocity $\hat{\omega}=[0,0, \omega]$.

The total mass of the body is $M$ while $\mathcal{G}$ is the universal gravitational constant and it is set $\mu=M \mathcal{G}$. The dynamics are formulated into a reference frame centered in the center of mass of the body and oriented with the " $z$ axis" parallel to the rotational axes of the asteroid. The frame of reference is rotating with the same velocity of rotation of the asteroid; in such rotating coordinates the Hamiltonian describing the system is:

$$
H(\mathbf{x}, \mathbf{X})=\frac{1}{2}(\mathbf{X} \cdot \mathbf{X})-\hat{\omega}(\mathbf{x} \times \mathbf{X})+\bar{U}(\mathbf{x})
$$

where $\mathbf{x}, \mathbf{X} \in \mathbb{R}^{3}$ are respectively the position coordinates and conjugate momenta of the spacecraft, while $\bar{U}(\mathbf{x})$ is the perturbing gravitational potential generated by the inhomogeneous rotating body. The equations of motion are:

$$
\left\{\begin{array}{l}
\dot{\mathbf{x}}=\frac{\partial}{\partial \mathbf{X}} H(\mathbf{x}, \mathbf{X}) \\
\dot{\mathbf{X}}=-\frac{\partial}{\partial \mathbf{x}} H(\mathbf{x}, \mathbf{X})
\end{array}\right.
$$

122

usi

to

six

126

27 8

It is convenient to express the Hamiltonian and the perturbing potential using the so called nodal-polar variables so that it may easily be transformed to the Delaunay coordinates in the later stage of the methodology. The six nodal-polar coordinates are $r, \theta$, and $\nu$ (respectively the distance of the spacecraft from the body, its angular distance from the line of the ascending node and the argument of node) and their corresponding conjugate momenta $R, \Theta$, and $N$. The transformation required is given in Palacián (2002), 
129

setting $\mathbf{x}=[x, y, z]^{T}$ and $\mathbf{X}=[X, Y, Z]^{T}$ :

$$
\begin{aligned}
& x=r(\cos \theta \cos \nu-\sin \theta \cos I \sin \nu) \\
& y=r(\cos \theta \sin \nu+\sin \theta \cos I \cos \nu) \\
& z=r \sin \theta \sin I \\
& X=\left(R \cos \theta-\frac{\Theta}{r} \sin \theta\right) \cos \nu-\left(R \sin \theta+\frac{\Theta}{r} \cos \theta\right) \cos I \sin \nu \\
& Y=\left(R \cos \theta-\frac{\Theta}{r} \sin \theta\right) \sin \nu+\left(R \sin \theta+\frac{\Theta}{r} \cos \theta\right) \cos I \cos \nu \\
& Z=\left(R \sin \theta+\frac{\Theta}{r} \cos \theta\right) \sin I
\end{aligned}
$$

130

135

136

with

$$
\begin{aligned}
& \mathcal{A}_{n, m, j, t}=\overline{\mathcal{G}}_{n, m, j, t}\left(C_{n, m} \cos \left(\frac{\pi}{2}(j+m)\right)-S_{n, m} \sin \left(\frac{\pi}{2}(j+m)\right)\right) \\
& \mathcal{B}_{n, m, j, t}=\overline{\mathcal{G}}_{n, m, j, t}\left(C_{n, m} \sin \left(\frac{\pi}{2}(j+m)\right)+S_{n, m} \cos \left(\frac{\pi}{2}(j+m)\right)\right),
\end{aligned}
$$


137

and

$$
\begin{aligned}
& \overline{\mathcal{G}}_{n, m, j, t}=(-1)^{m+3 t-j+1} \mu \alpha^{n} \frac{(n+m) !(n-j) !}{t !(n+j-t) !(n+m-t) !(t-m-j) !}(-1)^{\frac{n+j}{2}} \frac{1}{2^{n}} \frac{(n+j) !}{\left(\frac{n+j}{2}\right) !\left(\frac{n-j}{2}\right) !} . \\
& \quad \cdot\left((n+j)_{\text {mod }_{2}}-1\right) .
\end{aligned}
$$

In these $\alpha$ is a conventionally chosen reference radius, usually taken as the radius of the circumscribing sphere of the small body and $x_{\text {mody }_{y}}$ stands for the value of $x$ modulus $y$, i.e. the integer remainder of the division of $x$ by $y$. The $C_{n, m}$ and $S_{n, m}$ in (17) are called spherical harmonic coefficients, defined as, $\forall 0 \leq m \leq n$ :

$$
\begin{aligned}
& C_{n, m}=\frac{\left(2-\delta_{m, 0}\right)}{M} \frac{(n-m) !}{(n+m) !} \int_{V}\left(\frac{r^{\prime}}{\alpha}\right)^{n} P_{n, m}\left(\sin \delta^{\prime}\right) \cos \left(m \lambda^{\prime}\right) \rho\left(r^{\prime}, \delta^{\prime}, \lambda^{\prime}\right) d V \\
& S_{n, m}=\frac{\left(2-\delta_{m, 0}\right)}{M} \frac{(n-m) !}{(n+m) !} \int_{V}\left(\frac{r^{\prime}}{\alpha}\right)^{n} P_{n, m}\left(\sin \delta^{\prime}\right) \sin \left(m \lambda^{\prime}\right) \rho\left(r^{\prime}, \delta^{\prime}, \lambda^{\prime}\right) d V
\end{aligned}
$$

Where $\delta_{0, m}$ is the Kronecker delta that gives 1 if $m=0$, and 0 elsewhere, $P_{n}^{m}(x)$ is the associated Legendre function of degree $n$ and order $m$.

Moreover $r^{\prime} \in(0 ; \infty), \theta^{\prime} \in[-\pi ; \pi)$ and $\lambda^{\prime} \in[0 ; 2 \pi)$ are respectively the position, latitude and longitude of the infinitesimal volume element $d V$ in a cartesian frame of reference $O_{x, y, z}, \rho\left(r^{\prime}, \theta^{\prime}, \lambda^{\prime}\right)$ is the density of the infinitesimal element of volume and $V$ is the volume of the body.

Note that, in order to obtain formula (16) the gravitational potential

$$
U(r)=-\frac{\mathcal{G}}{r} \int_{V} \frac{\rho\left(r^{\prime}\right)}{\sqrt{1-2 \frac{r^{\prime}}{r} \cos \psi+\left(\frac{r^{\prime}}{r}\right)^{2}}} d V
$$

where $\cos \psi=\sin \delta \sin \delta^{\prime}+\cos \delta \cos \delta^{\prime} \cos \left(\lambda-\lambda^{\prime}\right)$, has been developed in terms of Legendre Polynomials using $\left(1-2 W Z+Z^{2}\right)^{-1 / 2}=\sum_{n=0}^{\infty} Z^{n} P_{n}(W)$. 
152

Thus it has been obtained that

$$
U(r)=-\frac{\mathcal{G}}{r} \int_{V} \sum_{n=0}^{\infty}\left(\frac{r^{\prime}}{r}\right)^{n} P_{n}(\cos \psi) \rho\left(r^{\prime}\right) d V,
$$

153 which converges only if the condition $\frac{r^{\prime}}{r}<1$ is satisfied, which implies that

the model is valid only outside the reference sphere.

A full explanation of the spherical harmonic coefficients can be found in Hofmann-Wellenhof et al. (1967). However it is important to highlight that equations in (9) imply:

$$
\begin{aligned}
& C_{0,0}=1 \\
& C_{n, 0}=\frac{1}{M} \int_{V}\left(\frac{r^{\prime}}{\alpha}\right)^{n} P_{n}\left(\sin \delta^{\prime}\right) \rho\left(r^{\prime}, \delta^{\prime}, \lambda^{\prime}\right) d V \quad \forall n>0 \\
& S_{n, 0}=0 \quad \forall n \geq 0
\end{aligned}
$$

Moreover, centering the origin of the system of reference at the center of mass it can be demonstrated that the term $C_{1,0}=0$.

The coefficients $C_{2,0}$ and $C_{2,2}$ express the "ellipticity" and "oblateness" of the body.

\section{The relegation of the polar component of the angular momen- $\operatorname{tum} N$}

In the context of artificial satellite theory, in general, one needs to order the terms of the Hamiltonian $H$ according to an asymptotic expansion in order to build a perturbation theory. The usual way to arrange the Hamiltonian for the cases in which the angular velocity of the asteroid is higher than the mean motion of the spacecraft (which holds, for example, for fast 
rotating bodies or for relatively high altitudes) is here followed (see Segerman and Coffey (2000)). It consists in placing the full unperturbed part at zeroth order and distribute the perturbation at first and second orders. The dominant (unperturbed) part of the Hamilton function is set to be the sum of the two-body Hamiltonian $H_{K}$ and the Coriolis term $H_{C}$. The perturbing potential takes into account an arbitrary number of spherical harmonic coefficients, distributed as first or second orders perturbations, depending on the harmonics of the specific asteroid studied, thus providing a dynamical model based on an arbitrarily accurate model of the inhomogeneous body.

The flows associated to the two components of the unperturbed Hamiltonian are used to relegate the whole system first and then to put it into normal form by means of symplectic transformations.

The Hamiltonian in (4) is therefore rearranged as:

$$
H=H_{0}+\epsilon H_{1}+\frac{\epsilon^{2}}{2} H_{2}+O\left(\epsilon^{3}\right)
$$

where $\epsilon$ is merely an ordering dimensionless parameter, which will be decided later on for the applications, and

$$
\begin{aligned}
& H_{0}=H_{K}+H_{C} \\
& H_{1}=U^{(1)}\left(r, \theta, \nu,{ }_{-}, \Theta, N\right) \\
& H_{2}=U^{(2)}\left(r, \theta, \nu,,_{-}, \Theta, N\right)
\end{aligned}
$$

where:

$$
\begin{aligned}
& H_{K}:=\frac{1}{2}\left(R^{2}+\frac{\Theta^{2}}{r^{2}}\right)-\frac{\mu}{r} \\
& H_{C}=-\omega N,
\end{aligned}
$$


185

and, for $s=1,2$

$$
\begin{gathered}
U^{(s)}\left(r, \theta, \nu,{ }_{-}, \Theta, N\right)=-\frac{s !}{\epsilon^{s}} \sum_{n=1}^{\infty} \sum_{m=0}^{n} \sum_{j=-n}^{n} \sum_{t=\max \{0, j+m\}}^{\min \{n+m, n+j\}} \mathrm{ci}^{2 \mathrm{n}+\mathrm{m}+\mathrm{j}-2 \mathrm{t}} \mathrm{si}^{2 \mathrm{t}-\mathrm{m}-\mathrm{j}} \\
\cdot \frac{1}{r^{n+1}}\left(\mathcal{A}_{n, m, j, t}^{(s)} \cos (m \nu-j \theta)+\mathcal{B}_{n, m, j, t}^{(s)} \sin (m \nu-j \theta)\right)
\end{gathered}
$$

186 with:

$$
\begin{aligned}
& \mathcal{A}_{n, m, j, t}^{(s)}=\overline{\mathcal{G}}_{n, m, j, t}\left(C_{n, m}^{(s)} \cos \left(\frac{\pi}{2}(j+m)\right)-S_{n, m}^{(s)} \sin \left(\frac{\pi}{2}(j+m)\right)\right) \\
& \mathcal{B}_{n, m, j, t}^{(s)}=\overline{\mathcal{G}}_{n, m, j, t}\left(C_{n, m}^{(s)} \sin \left(\frac{\pi}{2}(j+m)\right)+S_{n, m}^{(s)} \cos \left(\frac{\pi}{2}(j+m)\right)\right),
\end{aligned}
$$

187

with:

$$
\begin{aligned}
& C_{n, m}^{(s)}=\left\{\begin{array}{l}
C_{n, m} \text { if the term containing } C_{n, m} \text { is } \sim O\left(\epsilon^{q}\right) \\
0 \quad \text { otherwise }
\end{array}\right. \\
& S_{n, m}^{(s)}= \begin{cases}S_{n, m} \text { if the term containing } S_{n, m} \text { is of } \sim O\left(\epsilon^{q}\right) \\
0 \quad \text { otherwise }\end{cases}
\end{aligned}
$$

Again ci and si as in (6) and $\overline{\mathcal{G}}_{n, m, j, t}$ as in (8).

Now, considering the case $\left|H_{K}\right|<\left|H_{C}\right|$, two different Lie transformations are performed: the relegation of the polar component of the angular momentum $N$ first and the Delaunay normalisation.

Definition 1. A Lie transformation $\phi$ is a one-parameter family of mappings $\phi:(y, Y ; \epsilon) \rightarrow(x, X)$, defined by the solution $x(y, Y ; \epsilon)$ and $X(y, Y ; \epsilon)$ of the Hamiltonian system

$$
\left\{\begin{array}{l}
\frac{d x}{d \epsilon}=\frac{\partial W}{\partial X} \\
\frac{d X}{d \epsilon}=-\frac{\partial W}{\partial x}
\end{array}\right.
$$


with initial conditions $x(y, Y ; 0)=y$ and $X(y, Y ; 0)=Y$, and where the function

$$
W(x, X ; \epsilon)=\sum_{s \geq 0} \frac{\epsilon^{s}}{s !} W_{s+1}(x, X)
$$

193

is the generator of the transformation.

Due to the properties of the Hamiltonian systems, the Lie transformation $\phi$ is a completely canonical transformation that maps a Hamiltonian

$$
H(x, X ; \epsilon)=\sum_{s \geq 0} \frac{\epsilon^{s}}{s !} H_{s}(x, X)
$$

onto an equivalent Hamiltonian $K$ of the form

$$
K(y, Y ; \epsilon)=\sum_{s \geq 0} \frac{\epsilon^{s}}{s !} K_{s}(y, Y ; 0) .
$$

found by solving a series of homological equations:

$$
\left[H_{0} ; W_{s}\right]+\tilde{H}_{s}=K_{s} \quad \forall s \geq 1
$$

where the symbol [; ] stands for the Poisson Brackets. In equation (19) the element $\tilde{H}_{s}$ collects the terms from the previous orders (see Deprit (1969) and Palacián (2002)). The relegation and the normalization algorithms (see Deprit et al. (2001) and Deprit (1982) respectively) are two different methods of solving such homological equations. In particular, the relegation maps the Hamiltonian (13) into an equivalent one of the form:

$$
K=K_{0}+\sum_{s \geq 1} \frac{\epsilon^{s}}{s !} K_{s}=\sum_{s \geq 0} \frac{\epsilon^{s}}{s !}\left(\sum_{j=0}^{p} K_{s, p}+R_{s}\right)
$$

with $K_{0}=H_{0}(y, Y)$ and the coefficients $K_{s, p} \in \operatorname{ker}\left(\mathcal{L}_{H_{C}}\right)$, where $\mathcal{L}_{H_{C}}$ is the 
Lie derivative with respect to the Coriolis term ${ }^{1}$.

In contrast with normalization, the term $K_{s}$ may not belong to $\operatorname{ker}\left(\mathcal{L}_{H_{C}}\right)$ due to the presence of the residual $R_{s}$. In this resulting Hamiltonian the terms containing the variable $\nu$ will only appear in the remainder $R_{s}$. Moreover, for every order $s$ of the Hamiltonian, the algorithm iterated $p^{(s)}$ times (depending on the choice of the small parameter $\epsilon$ ), progressively diminishing the importance of the remainder $R_{s}$, such that after $p^{(s)}$ times it results $R_{s} \sim O\left(\epsilon^{3}\right)$

As a result the truncated system

$$
K=\sum_{s \geq 0} \frac{\epsilon^{s}}{s !} \sum_{j=0}^{p} K_{s, p}
$$

is obtained, which represents an approximation of the starting Hamiltonian independent from $\nu$ and admits $H_{C}$ as an integral.

In this section, in order to keep the generality of the analysis, the relegation is performed to the second order, arbitrary number of iterations $p^{(s)}$. In the applications section, once the parameter $\epsilon$ will be fixed, the number of iterations necessary to relegate the terms of the Hamiltonian containing $\nu$ to orders $\sim O\left(\epsilon^{3}\right)$ will therefore be estimated.

\footnotetext{
${ }^{1}$ Let $\mathcal{L}_{W}$ be the Lie derivative induced by the function $W$, then $\mathcal{L}_{W}$ which maps any function $f(X, x)$ into its Poisson Bracket with $W$, namely $f(X, x): \rightarrow[f ; W]$.

It must be noted that $\mathcal{L}_{H_{C}} H_{K}=0$ and that $\mathcal{L}_{H_{C}}$ is semi-simple over a Poisson algebra of functions $P$.
} 
218

219 220

228

229

230

\subsection{Algorithm}

The general relegation algorithm is briefly described here before the application to the problem. For each homological equation $(\forall s \geq 1)$ :

$$
\left[H_{0} ; W_{s}\right]+\tilde{H}_{s}=K_{s}
$$

considering that, as $\mathcal{L}_{H_{C}}$ is semi-simple, there $\exists K_{s, 0}, W_{s, 0} \in P$ s.t.

$$
\left\{\begin{array}{l}
\tilde{H}_{s}=K_{s, 0}+\left[W_{s, 0} ; H_{C}\right] \\
K_{s, 0} \in \operatorname{Ker}\left(\mathcal{L}_{\mathrm{H}_{\mathrm{C}}}\right) .
\end{array}\right.
$$

Therefore (22) becomes:

$$
\left[H_{0} ; W_{s}\right]+\left[W_{s, 0} ; H_{C}\right]=K_{s}-K_{s, 0} .
$$

Thus, setting $W_{s}=W_{s, 0}^{*}+W_{s, 0},(24)$ yields:

$$
\left[H_{0} ; W_{s}^{*}\right]+\left[H_{0}-H_{C} ; W_{s, 0}\right]=K_{s}-K_{s, 0} .
$$

The algorithm continues re-invoking $p^{(s)}$-times the semi-simplicity of $\mathcal{L}_{H_{C}}$, and finding $\forall 1 \leq p \leq p^{(s)} \quad K_{s, p}, W_{s, p} \in P$ s.t.

$$
\left\{\begin{array}{l}
{\left[H_{0}-H_{C} ; W_{s, p-1}\right]=K_{s, p}+\left[W_{s, p} ; H_{C}\right]} \\
K_{s, p} \in \operatorname{Ker}\left(\mathcal{L}_{\mathrm{H}_{\mathrm{C}}}\right)
\end{array}\right.
$$

$$
\text { and setting } p^{(s)} \text {-times } \forall 1 \leq p \leq p^{(s)} \quad W_{s, p-1}=W_{s, p}^{*}+W_{s, p} .
$$


231 232

234

235

$$
\begin{aligned}
W_{1,0}=-\frac{1}{\omega} \int\left(H_{1}-K_{1,0}\right) d \nu \\
=-\left(\frac{1}{\epsilon} \sum_{n=1}^{\infty} \sum_{m=1}^{n} \sum_{j=-n}^{n} \sum_{t=\max \{0, j+m\}}^{\min \{n+m, n+j\}} \mathrm{ci}^{2 \mathrm{n}+\mathrm{m}+\mathrm{j}-2 \mathrm{t}} \mathrm{si}^{2 \mathrm{t}-\mathrm{m}-\mathrm{j}}\left(-\frac{1}{\mathrm{~m} \omega}\right) \frac{1}{\mathrm{r}^{\mathrm{n}+1}}\right. \\
\left.\cdot\left(\mathcal{A}_{n, m, j, t}^{(1)} \sin (m \nu-j \theta)+\mathcal{B}_{n, m, j, t}^{(1)}(-\cos (m \nu-j \theta))\right)\right)
\end{aligned}
$$

with $R_{s}:=\left[H_{0}-H_{C} ; W_{s, p^{(s)}}\right]$.

Although the procedure is general, in view of the applications, only the first two homological equations will here be considered and explicitly solved.

\subsection{Results}

Following the procedure just described and Deprit (1969), for the first order $s=1$ of the Hamiltonian (13), we have that:

$$
\tilde{H}_{1,0}=H_{1}
$$

therefore, after the first iteration $p=1$, it results:

$$
\begin{aligned}
K_{1,0}=- & \frac{1}{\epsilon} \sum_{n=1}^{\infty} \sum_{j=-n}^{n} \sum_{t=\max \{0, j\}}^{\min \{n, n+j\}} \mathrm{ci}^{2 \mathrm{n}+\mathrm{j}-2 \mathrm{t}} \mathrm{si}^{2 \mathrm{t}-\mathrm{j}} \frac{1}{\mathrm{r}^{\mathrm{n}+1}}\left(\mathcal{A}_{\mathrm{n}, 0, \mathrm{j}, \mathrm{t}}^{(1)} \cos (-\mathrm{j} \theta)\right. \\
& \left.+\mathcal{B}_{n, 0, j, t}^{(1)} \sin (-j \theta)\right) .
\end{aligned}
$$


242

$$
\begin{aligned}
& {\left[H_{K}, W_{1,0}\right]=R \frac{\partial W_{1,0}}{\partial r}+\frac{\Theta}{r^{2}} \frac{\partial W_{1,0}}{\partial \theta}-\left(\frac{\Theta^{2}}{r^{3}}-\frac{M \mathcal{G}}{r^{2}}\right) \frac{\partial W_{1,0}}{\partial R}} \\
& =-\frac{1}{\epsilon} \sum_{n=1}^{\infty} \sum_{m=1}^{n} \sum_{j=-n}^{n} \sum_{t=\max \{0, j+m\}}^{\min \{n+m, n+j\}} \mathrm{ci}^{2 \mathrm{n}+\mathrm{m}+\mathrm{j}-2 \mathrm{t}} \mathrm{si}^{2 \mathrm{t}-\mathrm{m}-\mathrm{j}}\left(-\frac{1}{\mathrm{~m} \omega}\right)\left(-\frac{\mathrm{R}}{\mathrm{r}}\right) \cdot \\
& \cdot(-(n+1)) \frac{1}{r^{n+1}}\left(\mathcal{A}_{n, m, j, t}^{(1)} \sin (m \nu-j \theta)+\mathcal{B}_{n, m, j, t}^{(1)}(-\cos (m \nu-j \theta))\right) \\
& -\frac{1}{\epsilon} \sum_{n=1}^{\infty} \sum_{m=1}^{n} \sum_{j=-n}^{n} \sum_{t=\max \{0, j+m\}}^{\min \{n+m, n+j\}} \mathrm{ci}^{2 \mathrm{n}+\mathrm{m}+\mathrm{j}-2 \mathrm{t}} \mathrm{si}^{2 \mathrm{t}-\mathrm{m}-\mathrm{j}}\left(-\frac{1}{\mathrm{~m} \omega}\right)\left(\frac{\mathrm{j} \Theta}{\mathrm{r}^{2}}\right) \frac{1}{\mathrm{r}^{\mathrm{n}+1}} \cdot \\
& \cdot\left(\mathcal{A}_{n, m, j, t}^{(1)} \cos (m \nu-j \theta)+\mathcal{B}_{n, m, j, t}^{(1)} \sin (m \nu-j \theta)\right),
\end{aligned}
$$

${ }_{243}$ Then the algorithm is iterated $\forall 1<p \leq p^{(s)}$, where at each iteration it 244 results:

$$
K_{1, p}=0
$$

245 Calling $p_{O_{\max }}=2\left\lfloor\frac{p-1}{2}\right\rfloor+1, \quad p_{E_{\max }}=2\left\lfloor\frac{p}{2}\right\rfloor$, and:

$$
\begin{aligned}
& \mathcal{S}\left(\hat{k}, k^{*}\right)=\sum_{k=\hat{k}}^{k^{*}} a_{k} \\
& \mathcal{S}_{E}\left(\hat{k}, k^{*}\right)=\sum_{k=\hat{k},}^{k^{*}} a_{k}, \quad \mathcal{S}_{E}^{\prime}\left(\hat{k}, k^{*}\right)=\sum_{k=\hat{k},}^{k^{*}} a_{k}^{\prime}, \quad \mathcal{S}_{E}^{\prime \prime}\left(\hat{k}, k^{*}\right)=\sum_{k=\hat{k},}^{k^{*}} a_{k}^{\prime \prime} \\
& k \text { even } k \text { even } \quad k \text { even } \\
& \mathcal{S}_{O}\left(\hat{k}, k^{*}\right)=\sum_{\substack{k=\hat{k}, k \text { odd }}}^{k^{*}} a_{k}, \quad \mathcal{S}_{O}^{\prime}\left(\hat{k}, k^{*}\right)=\sum_{\substack{k=\hat{k}, k \text { odd }}}^{k^{*}} a_{k}^{\prime}, \quad \mathcal{S}_{O}^{\prime \prime}\left(\hat{k}, k^{*}\right)=\sum_{\substack{k=\hat{k}, k \text { odd }}}^{k^{*}} a_{k}^{\prime \prime}
\end{aligned}
$$

246 Also, calling:

$$
\mathcal{D}:=(-1)^{p-\mathcal{S}(1, p)}\left(\begin{array}{l}
p-\mathcal{S}(2, p) \\
p-\mathcal{S}(1, p)
\end{array}\right) \frac{(n+p-\mathcal{S}(1, p)) !}{\left(n+a_{1}\right) !}
$$

247 and $\forall k$ odd 


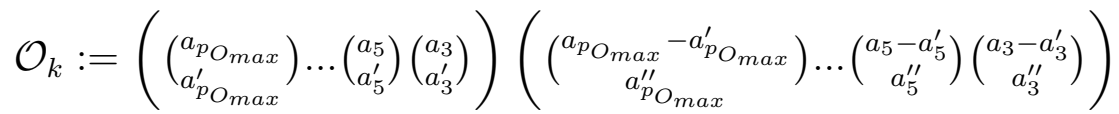

$$
\begin{aligned}
& \left(\frac{\left(a_{1}+n+p+2 \mathcal{S}_{E}(2, k-1)-\mathcal{S}(k, p)+a_{k}-a_{k}^{\prime \prime}+\mathcal{S}_{O}^{\prime}(3, k-2)-\mathcal{S}_{O}^{\prime \prime}(3, k-2)\right) !}{\left(a_{1}+n+p+2 \mathcal{S}_{E}(2, k-1)-\mathcal{S}(k, p)+a_{k}^{\prime}+\mathcal{S}_{O}^{\prime}(3, k-2)-\mathcal{S}_{O}^{\prime \prime}(3, k-2)\right) !}\right) \\
& \left(\frac{\left(a_{1}+n+p+\mathcal{S}_{E}(2, k-1)-\mathcal{S}(k, p)+a_{k}+\mathcal{S}_{O}(3, k-2)-\mathcal{S}_{O}^{\prime \prime}(3, k-2)\right) !}{\left(a_{1}+n+p+\mathcal{S}_{E}(2, k-1)-\mathcal{S}(k, p)+a_{k}-a_{k}^{\prime \prime}+\mathcal{S}_{O}(3, k-2)-\mathcal{S}_{O}^{\prime \prime}(3, k-2)\right) !}\right)
\end{aligned}
$$

$248 \quad$ while $\forall k$ even

$$
\begin{aligned}
& \mathcal{E}_{2}:=\left(\frac{(p-\mathcal{S}(1, p)) !}{\left(p-\mathcal{S}(1, p)-a_{2}\right) !}\right) \\
& \mathcal{E}_{k}:=\left(\frac{\left(p-a_{1}-2 \mathcal{S}_{E}(2, k-2)-\mathcal{S}(k, p)-\mathcal{S}_{O}^{\prime}(3, k-1)+a_{k}+1\right) !}{\left(p-a_{1}-2 \mathcal{S}_{E}(2, k-2)-\mathcal{S}(k, p)-\mathcal{S}_{O}^{\prime}(3, k-1)-1\right) !}\right) \quad \forall k \geq 4, \quad k \text { even }
\end{aligned}
$$


it results:

$$
\begin{aligned}
& W_{1, p}=-\frac{1}{\epsilon} \sum_{n=1}^{\infty} \sum_{m=1}^{n} \sum_{j=-n}^{n} \sum_{t=\max \{0, j+m\}}^{\min \{n+m, n+j\}} \mathrm{ci}^{2 \mathrm{n}+\mathrm{m}+\mathrm{j}-2 \mathrm{t}} \mathrm{si}^{2 \mathrm{t}-\mathrm{m}-\mathrm{j}}\left(-\frac{1}{\mathrm{~m} \omega}\right)^{\mathrm{p}+1} \\
& \sum_{a_{p}=0}^{1}\left(\sum _ { a _ { p - 1 } = 1 - \delta _ { a _ { p } , 0 } } ^ { \operatorname { m a x } \{ p - ( p - 2 ) , 0 \} } \sum _ { a _ { 3 } = 1 - \delta _ { a _ { 4 } , 0 } } ^ { \operatorname { m a x } \{ p - \mathcal { S } ( 4 , p ) - 2 , 0 \} } \sum _ { a _ { 2 } = 1 - \delta _ { a _ { 3 } , 0 } } ^ { \operatorname { m a x } \{ p - \mathcal { S } ( 3 , p ) - 1 , 0 \} } \left(\sum_{a_{1}=0}^{\max \{p-\mathcal{S}(2, p), 0\}}\right.\right.
\end{aligned}
$$

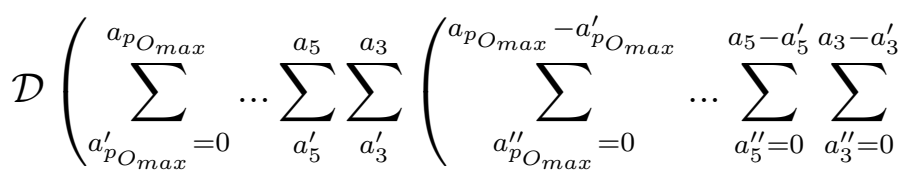

$$
\begin{aligned}
& \left(\mathcal{O}_{p_{O_{\max }}} \cdot \ldots \cdot \mathcal{O}_{5} \cdot \mathcal{O}_{3}\right)\left(\mathcal{E}_{p_{E_{\max }}} \cdot \ldots \cdot \mathcal{E}_{4} \mathcal{E}_{2}\right)\left(\frac{1}{r}\right)^{3\left(\mathcal{S}_{O}\left(3, p_{O_{\max }}\right)-\mathcal{S}_{O}^{\prime}\left(3, p_{O_{\text {max }}}\right)\right)} \\
& \left(-\frac{1}{r}\right)^{p-a_{1}-\mathcal{S}_{E}\left(2, p_{E_{\max }}\right)-\mathcal{S}_{O}^{\prime}\left(3, p_{O \max }\right)} R^{p-a_{1}-2 \mathcal{S}_{E}\left(2, p_{E_{\max }}\right)-\mathcal{S}_{O}^{\prime}\left(3, p_{O_{\max }}\right)} \\
& \left(\frac{j \Theta}{r^{2}}\right)^{a_{1}+\mathcal{S}_{O}^{\prime}\left(3, p_{O_{\max }}\right)}\left(\frac{-\Theta^{2}+r \mu}{r^{3}}\right)^{\mathcal{S}_{E}\left(2, p_{E_{\max }}\right)-\mathcal{S}_{O}\left(3, p_{O_{\max }}\right)+\mathcal{S}_{O}^{\prime}\left(3, p_{O_{\max }}\right)} \\
& \Theta^{2\left(\mathcal{S}_{O}\left(3, p_{\text {Omax }}\right)-\mathcal{S}_{O}^{\prime}\left(3, p_{O_{\max }}\right)-\mathcal{S}_{O}^{\prime \prime}\left(3, p_{O_{\max }}\right)\right)}(-r \mu)^{\mathcal{S}_{O}^{\prime \prime}\left(3, p_{O_{\max }}\right)} \frac{1}{r^{n+1}} \\
& \left(\mathcal { A } _ { n , m , j , t } ^ { ( 1 ) } \left(\cos (m \nu-j \theta) \cos \left(\frac{\pi}{2}\left(-(p+1)+a_{1}+\mathcal{S}_{O}^{\prime}\left(3, p_{O_{\max }}\right)\right)\right)\right.\right. \\
& \left.-\sin (m \nu-j \theta) \sin \left(\frac{\pi}{2}\left(-(p+1)+a_{1}+\mathcal{S}_{O}^{\prime}\left(3, p_{O_{\max }}\right)\right)\right)\right) \\
& +\mathcal{B}_{n, m, j, t}^{(1)}\left(\sin (m \nu-j \theta) \cos \left(\frac{\pi}{2}\left(-(p+1)+a_{1}+\mathcal{S}_{O}^{\prime}\left(3, p_{O_{\max }}\right)\right)\right)\right. \\
& \left.\left.\left.\left.\left.\left.+\cos (m \nu-j \theta) \sin \left(\frac{\pi}{2}\left(-(p+1)+a_{1}+\mathcal{S}_{O}^{\prime}\left(3, p_{O_{\max }}\right)\right)\right)\right)\right)\right)\right)\right)\right)
\end{aligned}
$$


and

$$
\begin{aligned}
& {\left[H_{K}, W_{1, p}\right]=-\frac{1}{\epsilon} \sum_{n=1}^{\infty} \sum_{m=1}^{n} \sum_{j=-n}^{n} \sum_{t=\max \{0, j+m\}}^{\min \{n+m, n+j\}} \mathrm{ci}^{2 \mathrm{n}+\mathrm{m}+\mathrm{j}-2 \mathrm{t}} \mathrm{si}^{2 \mathrm{t}-\mathrm{m}-\mathrm{j}}} \\
& \sum_{a_{p+1}=0}^{1}\left(\sum_{a_{p}=1-\delta_{a_{p+1}, 0}}^{\max \{p+1-(p-1), 0\}} \sum_{a_{3}=1-\delta_{a_{4}, 0}}^{\max \{p+1-\mathcal{S}(4, p+1)-2,0\}} \sum_{a_{2}=1-\delta_{a_{3}, 0}}^{\max \{p+1-\mathcal{S}(3, p+1)-1,0\}}\right. \\
& \left(\sum _ { a _ { 1 } = 0 } ^ { \operatorname { m a x } \{ p + 1 - \mathcal { S } ( 2 , p + 1 ) , 0 \} } \mathcal { D } ^ { * } \left(\sum_{a_{p_{O_{\max }}^{\prime}}=0}^{a_{p_{O_{\max }}+1}} \ldots \sum_{a_{5}^{\prime}}^{a_{5}} \sum_{a_{3}^{\prime}}^{a_{3}}\left(-\frac{1}{m \omega}\right)^{p+1}\right.\right.
\end{aligned}
$$

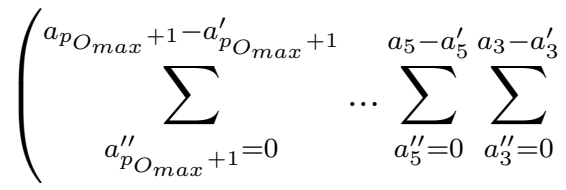

$$
\begin{aligned}
& \left(\mathcal{O}_{p_{O_{\max }+1}^{*}} \cdot \ldots \cdot \mathcal{O}_{5}^{*} \cdot \mathcal{O}_{3}^{*}\right)\left(\mathcal{E}_{p_{E \max }+1}^{*} \cdot \ldots \cdot \mathcal{E}_{4}^{*} \mathcal{E}_{2}^{*}\right) \\
& \left(\frac{1}{r}\right)^{3\left(\mathcal{S}_{O}\left(3, p_{O_{\max }}+1\right)-\mathcal{S}_{O}^{\prime}\left(3, p_{O_{\max }}+1\right)\right)} \\
& \left(-\frac{1}{r}\right)^{p+1-a_{1}-\mathcal{S}_{E}\left(2, p_{E_{\max }}+1\right)-\mathcal{S}_{O}^{\prime}\left(3, p_{O \max }+1\right)} \\
& R^{p+1-a_{1}-2 \mathcal{S}_{E}\left(2, p_{E_{\max }}+1\right)-\mathcal{S}_{O}^{\prime}\left(3, p_{O_{\max }}+1\right)} \\
& \left(\frac{j \Theta}{r^{2}}\right)^{a_{1}+\mathcal{S}_{O}^{\prime}\left(3, p_{O_{\text {max }}}+1\right)}\left(\frac{-\Theta^{2}+r \mu}{r^{3}}\right)^{\mathcal{S}_{E}\left(2, p_{E \max }+1\right)-\mathcal{S}_{O}\left(3, p_{O_{\max }}+1\right)+\mathcal{S}_{O}^{\prime}\left(3, p_{O_{\max }}+1\right)} \\
& \Theta^{2\left(\mathcal{S}_{O}\left(3, p_{O_{\max }}+1\right)-\mathcal{S}_{O}^{\prime}\left(3, p_{O_{\max }}+1\right)-\mathcal{S}_{O}^{\prime \prime}\left(3, p_{O_{\max }}+1\right)\right)}(-r \mu)_{\mathcal{S}_{O}^{\prime \prime}\left(3, p_{O_{\max }}+1\right)} \frac{1}{r^{n+1}}
\end{aligned}
$$




$$
\begin{aligned}
& \left(\mathcal { A } _ { n , m , j , t } ^ { ( 1 ) } \left(\cos (m \nu-j \theta) \cos \left(\frac{\pi}{2}\left(-(p+1)+a_{1}+\mathcal{S}_{O}^{\prime}\left(3, p_{O_{\max }}\right)\right)\right)\right.\right. \\
& \left.-\sin (m \nu-j \theta) \sin \left(\frac{\pi}{2}\left(-(p+1)+a_{1}+\mathcal{S}_{O}^{\prime}\left(3, p_{O_{\max }}\right)\right)\right)\right) \\
& +\mathcal{B}_{n, m, j, t}^{(1)}\left(\sin (m \nu-j \theta) \cos \left(\frac{\pi}{2}\left(-(p+1)+a_{1}+\mathcal{S}_{O}^{\prime}\left(3, p_{O_{\max }}\right)\right)\right)\right. \\
& \left.\left.\left.\left.\left.+\cos (m \nu-j \theta) \sin \left(\frac{\pi}{2}\left(-(p+1)+a_{1}+\mathcal{S}_{O}^{\prime}\left(3, p_{O_{\max }}\right)\right)\right)\right)\right)\right)\right)\right)
\end{aligned}
$$

where $\mathcal{D}^{*}, \mathcal{O}_{t}^{*}$ and $\mathcal{E}_{t}^{*}$ are like the one in (34), (35) and (36) respectively with $p+1$ instead of $p$.

As

$$
\left[H_{K} ; \cdot\right]=\left[\frac{1}{2}\left(R^{2}+\frac{\Theta^{2}}{r^{2}}\right)-\frac{\mu}{r} ; \cdot\right]=R \frac{\partial \cdot}{\partial r}+\frac{\Theta}{r^{2}} \frac{\partial \cdot}{\partial \theta}-\left(\frac{\Theta^{2}}{r^{3}}-\frac{\mu}{r^{2}}\right) \frac{\partial \cdot}{\partial R}
$$

at each step $p$ the term $\left[H_{K}, W_{1, p}\right]$ is the sum functions that have the same order of the preceding $\left[H_{K}, W_{1, p-1}\right]$ but multiplied by $\frac{R}{\omega r}, \frac{\Theta}{\omega r^{2}}$ or $\frac{\Theta^{2}+r}{\omega R r^{3}}$. Following Segerman and Coffey (2000), as $R \sim \frac{\Theta}{r}$ and as, at order zero, for the two-body problem, $\Theta \sim r^{2} \dot{\theta}$, for a satellite period greater than the rotational period of the asteroid (i.e. $\dot{\theta}<\omega$ ), and therefore these coefficients are less than unity over an orbit $\sim \frac{\dot{\theta}}{\omega}<1$. Therefore, at each step of the relegation, the transformation process reduces the magnitude of the terms of the perturbing potential which contain the angle $\nu$. Thus, after fixing the parameter $\epsilon$, the number of iteration $p^{(1)}$ is fixed such that $\left[H_{K}, W_{1, p^{(1)}}\right] \sim O\left(\epsilon^{3}\right)$.

The relegation of the first order is ended setting:

$$
\begin{aligned}
W_{1} & :=\sum_{p=0}^{p^{(1)}} W_{1, p} \\
R_{1} & :=\left[H_{K}, W_{1, p^{(1)}}\right] \\
K_{1} & :=\sum_{p=0}^{p^{(1)}} K_{1, p}+R_{1}=K_{1,0}+R_{1}
\end{aligned}
$$

To pass to the second order $s=2$, the evaluation of $\tilde{H}_{2,0}=H_{2}+$ $2\left[H_{1}, W_{1}\right]+\left[\left[H_{0}, W_{1}\right], W_{1}\right]$ is first required, from which the expression for 
$K_{2,0}$ is derived (see the Electronic Supplementary Material). In analogy with the first order, it results $K_{2, p}=0 \quad \forall p \geq 1$.

The relegation of the second order is ended setting:

$$
\begin{aligned}
& W_{2}:=\sum_{p=0}^{p^{(2)}} W_{2, p} \\
& R_{2}:=\left[H_{K}, W_{2, p^{(2)}}\right] \\
& K_{2}:=\sum_{p=0}^{p^{(2)}} K_{2, p}+R_{2}=K_{2,0}+R_{2}
\end{aligned}
$$

where $p^{(2)}$ is chosen such that $\left[H_{K}, W_{2, p^{(2)}}\right] \sim O\left(\epsilon^{3}\right)$ which is $p^{(2)}=\left\lfloor\frac{p^{(1)}+1}{2}\right\rfloor$.

The resulting Hamiltonian $K=K_{0}+\epsilon K_{1}+\frac{\epsilon^{2}}{2} K_{2}$ is completely equivalent to the one in (13). However, as the terms $R_{s}, s=1,2$ are of order $\sim \epsilon^{3}$, a truncated system is considered in which such terms have been neglected. Setting:

$$
\begin{aligned}
\tilde{K}_{0} & :=K_{0} \\
\tilde{K}_{1} & :=\sum_{p=0}^{p^{(1)}} K_{1, p}=K_{1,0} \\
\tilde{K}_{2} & :=\sum_{p=0}^{p^{(2)}} K_{2, p}=K_{2,0}
\end{aligned}
$$

the truncated system is described by the Hamiltonian:

$$
\tilde{K}=\tilde{K}_{0}+\epsilon \tilde{K}_{1}+\frac{\epsilon^{2}}{2} \tilde{K}_{2}
$$

where, to simplify notation, the ${ }^{\sim}$ will be ignored. This Hamiltonian is equivalent to the one in the main problem of the artificial satellite, in which the argument of node $\nu$ is cyclic, which implies that the coriolis term $-\omega N$ is constant and can therefore be dropped from the Hamiltonian. A closed form Delaunay normalization can now be performed, for a further reduction of the degrees of freedom, thus yielding an integrable Hamiltonian. 
It must be noted that, in complete analogy with the procedure adopted so far, the explicit formulation for every higher order $s \geq 2$ could be obtained.

\section{Delaunay Normalization}

In order to perform the Delaunay normalisation the Hamiltonian is transformed from the relegated Whittaker variables to the Delaunay coordinates. The Delaunay coordinates are symplectic action-angle variables $(L, G, H, \ell, g, h)$, where the angles $\ell, g$ and $h$ are conjugate to the actions $L, G$ and $H$ respectively. Among the angle variables $\ell$ is the mean anomaly measured from the pericenter, $g$ is the argument of the pericenter while $h$ is the argument of the node. For the actions instead $L$ is related to the major semi-axis, $a$, by $L=\sqrt{\mu a}, G$ is the total angular momentum of the spacecraft with respect to the Asteroid (in the inertial frame), related to the eccentricity and the variable $L$ by $e=\sqrt{1-\frac{G^{2}}{L^{2}}}$, and $H$ is the $z$-component of the total angular momentum, i.e. $H=G \cos I$.

The relation between the True anomaly and the Eccentric anomaly $u$ is defined as $\tan \left(\frac{f}{2}\right)=\sqrt{\frac{1+e}{1-e}} \tan \left(\frac{u}{2}\right)$, which, in particular, implies $r=a(1-$ $e \cos u)=a \frac{1-e^{2}}{1+e \cos f}$.

Moreover, by Section 3, we know that $N=G \cos I \Rightarrow H=G \cos I$ and $R=\frac{\mu e \sin f}{G}$.

The relegated Hamiltonian (43) in the Delaunay coordinates takes the form:

$$
J=J_{0}+\epsilon J_{1}+\frac{\epsilon^{2}}{2} J_{2}
$$


304

with:

$$
\begin{aligned}
J_{0}= & -\frac{(\mathcal{G} M)^{2}}{2 L^{2}} \\
J_{1}= & -\frac{1}{\epsilon} \sum_{n=1}^{\infty} \sum_{j=-n}^{n} \sum_{t=\max \{0, j\}}^{\min \{n, n+j\}} \mathrm{ci}^{2 \mathrm{n}+\mathrm{j}-2 \mathrm{t}} \mathrm{si}^{2 \mathrm{t}-\mathrm{j}}\left(\frac{(1+\mathrm{e} \cos \mathrm{f})}{\left(\mathrm{a}\left(1-\mathrm{e}^{2}\right)\right)}\right)^{\mathrm{n}+1} \\
& \left(\mathcal{A}_{n, 0, j, t}^{(1)} \cos (-j(f+g))+\mathcal{B}_{n, 0, j, t}^{(1)} \sin (-j(f+g))\right) .
\end{aligned}
$$

305

with

$$
\begin{aligned}
& c i=\sqrt{\frac{1+\frac{H}{G}}{2}} \\
& s i=\sqrt{\frac{1-\frac{H}{G}}{2}}
\end{aligned}
$$

In our case, as $K_{0}^{\prime}=J_{0}=-\frac{(\mathcal{G} M)^{2}}{2 L^{2}}$, the Lie derivative

$$
L_{K_{0}^{\prime}}(\cdot)=\frac{(\mathcal{G} M)^{2}}{L^{3}} \frac{\partial(\cdot)}{\partial \ell}
$$


therefore the new Hamiltonian (44) will be in normal form if and only if

$$
\frac{\partial K_{1}^{\prime}}{\partial \ell}=0 \text { and } \frac{\partial K_{2}^{\prime}}{\partial \ell}=0
$$

319

322

323

Note that, as for the relegation for the angle $\nu$, the normalization degenerates into an average over the mean anomaly $\ell$. Moreover it will be used that:

$$
\frac{d f}{d \ell}=\frac{a^{2} \sqrt{1-e^{2}}}{r^{2}} .
$$

\subsection{Results}

The explicit formula for the normalized $J_{1}$ is:

$$
\begin{aligned}
& K_{1}^{\prime}=-\frac{1}{\epsilon} \sum_{n=1}^{\infty} \sum_{t=\max \{0, j\}}^{\min \{n, n+j\}} \mathrm{ci}^{2 \mathrm{n}+\mathrm{j}-2 \mathrm{t}} \mathrm{si}^{2 \mathrm{t}-\mathrm{j}} \\
& \frac{\sqrt{1-e^{2}}}{a^{n+1}\left(1-e^{2}\right)^{n}}\left(\sum_{k=0}^{n-1}\left(\begin{array}{c}
n-1 \\
k
\end{array}\right) e^{k} \mathcal{A}_{n, 0, j, t}^{(1)} \frac{(k-1) ! !}{k ! !}(k+1)_{\text {mod }_{2}}+\right. \\
& \quad+2(n+1)_{\bmod _{2}} \sum_{j=1}^{n} \sum_{k=0}^{n-1} \sum_{q=0}^{\left\lfloor\frac{j}{2}\right\rfloor} \sum_{v=0}^{q}\left(\begin{array}{c}
n-1 \\
k
\end{array}\right)\left(\begin{array}{c}
j \\
2 q
\end{array}\right)\left(\begin{array}{c}
q \\
v
\end{array}\right)(-1)^{q+v} e^{k} \mathcal{A}_{n, 0, j, t}^{(1)} . \\
& \quad \cos (g j) \frac{((j-2 q+k+2 v)-1) ! !}{(j-2 q+k+2 v) ! !}((j-2 q+k+2 v)+1)_{\text {mod }_{2}} \\
& \quad-2(n)_{\bmod _{2}} \sum_{j=1}^{n} \sum_{k=0}^{n-1} \sum_{q=0}^{\left\lfloor\frac{j}{2}\right\rfloor} \sum_{v=0}^{q}\left(\begin{array}{c}
n-1 \\
k
\end{array}\right)\left(\begin{array}{c}
j \\
2 q
\end{array}\right)\left(\begin{array}{c}
q \\
v
\end{array}\right)(-1)^{q+v} e^{k} \mathcal{B}_{n, 0, j, t}^{(1)} . \\
& \left.\cdot \sin (g j) \frac{((j-2 q+k+2 v)-1) ! !}{(j-2 q+k+2 v) ! !}((j-2 q+k+2 v)+1)_{\text {mod }_{2}}\right)
\end{aligned}
$$

26 obtained using that, $\forall 1 \leq j \leq n$ :

$$
\begin{aligned}
& \text { if } n \text { even } \mathcal{A}_{n, 0, j}^{(1)}=\mathcal{A}_{n, 0,-j}^{(1)} \\
& \text { if } n \text { odd } \mathcal{A}_{n, 0, j}^{(1)}=-\mathcal{A}_{n, 0,-j}^{(1)}
\end{aligned}
$$


327

$$
\begin{aligned}
& \text { if } n \text { even } \mathcal{B}_{n, 0, j}^{(1)}=\mathcal{B}_{n, 0,-j}^{(1)} \\
& \text { if } n \text { odd } \mathcal{B}_{n, 0, j}^{(1)}=-\mathcal{B}_{n, 0,-j}^{(1)}
\end{aligned}
$$

The first order generating function is obtained by:

$$
W_{1}^{\prime}=\int \frac{L^{3}}{(\mu)^{2}}\left(J_{1}-\frac{1}{2 \pi} \int_{0}^{2 \pi} J_{1} d \ell\right) d \ell
$$

Finally the normalised $J_{2}$, namely

$$
K_{2}^{\prime}=\frac{1}{2 \pi} \int_{0}^{2 \pi}\left(J_{2}+2\left[J_{1}, W_{1}^{\prime}\right]+\left[\left[J_{0}, W_{1}^{\prime}\right], W_{1}^{\prime}\right]\right) d \ell
$$

and its corresponding generating function

$$
W_{2}^{\prime}=\int \frac{L^{3}}{(\mu)^{2}}\left(J_{2}-K_{2}^{\prime}\right) d \ell
$$

have been evaluated, using integration by parts, with the aid the software Mathematica.

As a result $K^{\prime}=K_{0}^{\prime}+\epsilon K_{1}^{\prime}+\frac{\epsilon^{2}}{2} K_{2}^{\prime}$ is obtained which is the analytical formulation for the closed-form averaged (with respect to both the argument of node and the mean anomaly), second order, arbitrary degree Hamiltonian of any inhomogeneous gravitational field of a body uniformly rotating around its main axes of inertia for the case $\left|H_{K}\right|<\left|H_{C}\right|$. This two degree of freedom, integrable Hamiltonian approximates the initial system, and can now be applied to every inhomogeneous body in order to determine possible orbits useful for scientific observation missions such as frozen orbits. 


\section{Applications}

The Hamiltonian obtained is of the form: $K^{\prime}\left(L, G, H,{ }_{-}, g,_{-}\right)$thus the equations of motion are:

$$
\begin{aligned}
\ell^{\prime}(t) & =\frac{\partial K^{\prime}}{\partial L} \\
g^{\prime}(t) & =\frac{\partial K^{\prime}}{\partial G} \\
h^{\prime}(t) & =\frac{\partial K^{\prime}}{\partial H} \\
L^{\prime}(t) & =0 \\
G^{\prime}(t) & =-\frac{\partial K^{\prime}}{\partial g} \\
H^{\prime}(t) & =0,
\end{aligned}
$$

which can be derived by (48) and (52) where $L$ and $H$ are constants and all the other motions will only depend on $G(t)$ and $g(t)$.

\section{Definition 3. (Frozen orbit)}

A frozen orbit is an orbit in which the Inclination, the Eccentricity and the Argument of pericenter remains constant during the motion.

This in particular implies that such an orbit is then perfectly periodic except for the orbital plane precession.

A frozen orbit it thus described by the system:

$$
\begin{aligned}
\dot{e} & =\frac{d}{d t} \frac{\sqrt{L^{2}-G^{2}}}{L}=0 \\
\dot{I} & =\frac{d}{d t} \arccos \frac{H}{G}=0 \\
\dot{g} & =0 .
\end{aligned}
$$

For the properties of the Lie transformations, the "normalized" eccentricity, inclination and argument of pericenter are related to their relative "real" equivalents by the generator of the transformation (see Deprit (1969)), and 
can thus be interpreted as a perturbed version of their real correspondents.

In the normalized variables (54), the system (55) is equivalent to:

$$
\begin{aligned}
& \dot{G}=0 \\
& \dot{g}=0 .
\end{aligned}
$$

Thus fixing normalized eccentricity $e$ and inclination $I$ for the desired normalized frozen orbit, and solving the system gives:

$$
\begin{aligned}
& \dot{G}=0 \\
& \dot{g}=0 \\
& e=\frac{\sqrt{L^{2}-G^{2}}}{L} \\
& I=\arccos \frac{H}{G},
\end{aligned}
$$

be found.

Moreover, as this all procedure is valid for the case $\left|H_{K}\right|<\left|H_{C}\right|$ such initial conditions must satisfy:

$$
\omega H_{0}>\frac{\mu^{2}}{2 L_{0}^{2}}
$$

and also

$$
0<\left|H_{0}\right|<G_{0}<L_{0}
$$

These resulting initial conditions can transformed back to the initial system describing the full dynamics (see (13)) by the inverse of the generating functions (Deprit (1969)), to generate an initial guess for frozen orbits around any inhomogeneous body.

\section{Conclusions}

Setting the desired eccentricity and inclination it is thus possible to determine initial conditions which lead to frozen orbits in the truncated system. 
Such initial conditions are used to approximate the solutions for the secular motion of the satellite in the real system thus showing a good agreement between the approximated and the real dynamics.

An example of the application of the method is shown for the asteroid 433Eros, a highly irregular, elongated, Near Earth Asteroid, which is the main example used in the literature, for which the spherical harmonic coefficients up to the $15^{t h}$ order and degree (i.e.272 coefficients) are listed in the Appendix A.

The physical properties of this asteroid are summarized in the table (1).

\begin{tabular}{|c|c|c|c|}
\hline & Mass & Rotational velocity & Reference Radius \\
$\mathrm{Kg}$ & $\mathrm{rad} / \mathrm{s}$ & $\mathrm{Km}$ \\
\hline 433-Eros & $6.6904 \times 10^{15}$ & $3.31182 \times 10^{-4}$ & 16 \\
\hline
\end{tabular}

Table 1: Physical properties of 433-Eros

In inverse analogy with Palacián (2002) we would like to take $\epsilon \sim \frac{\mu^{2}}{\omega L_{0}^{3}}$. Considering the resulting frozen orbits to be at an altitude high enough to satisfy the condition $\left|H_{K}\right|>\left|H_{C}\right|$, and trying to include an high number of spherical harmonic coefficients in the model, in the example shown the ordering parameter $\epsilon$ is set to be $\epsilon=10^{-2}$ (i.e. semimajor axes $\sim 300 \mathrm{~km}$, $p^{(1)}=2, p^{(2)}=2$ s.t. $\left.R_{1} \sim\left(\frac{\dot{\theta}}{\omega}\right)^{p^{(1)}+1} \sim 10^{-6}\right)$.

For this example the numerical estimation of the terms containing 433-Eros' spherical Harmonics up to order and degree 15, leads to the distribution of the $C_{n, m}, S_{n, m}$ between the $C_{n, m}^{(1)}, C_{n, m}^{(2)}$ and the $S_{n, m}^{(1)}, S_{n, m}^{(2)}$ respectively. 
392

For the result shown below it will thus be fixed that:

$$
\begin{aligned}
& C_{n, m}^{(1)}=\left\{\begin{array}{l}
C_{n, m} \text { if }(n, m) \in\{(0,0),(2,0),(2,2)\} \\
0 \text { otherwise }
\end{array}\right. \\
& C_{n, m}^{(2)}=\left\{\begin{array}{l}
C_{n, m} \text { if }(n, m) \in\{(3,1),(3,3),(4,0),(4,2),(4,4),(5,1), \\
0 \text { otherwise }
\end{array}\right. \\
& S_{n, m}^{(1)}=\left\{\begin{array}{l}
S_{n, m} \text { if }(n, m) \in\{(2,2)\} \\
0 \text { otherwise } \\
S_{n, m} \text { if }(n, m) \in\{(3,1),(3,3),(6,2),(6,6)\} \\
0 \text { otherwise }
\end{array}\right. \\
& S_{n, m}^{(2)}=\left\{\begin{array}{l}
0,2),(4,4),(5,3),(5,5)\}
\end{array}\right.
\end{aligned}
$$

For illustration purposes the initial eccentricity has been set to $E_{0}=$ 0.5, the inclination to $I_{0}=1.1$ and argument of pericenter to $g_{0}=-\frac{\pi}{2}$, yielding to the initial conditions $f_{0}, h_{0}, L_{0}, G_{0}$, and $H_{0}$ for the (relegated and normalized) frozen orbit collected in Table (2) for 433-Eros. In the last row of the table, the initial semimajor axes $a_{0}$ of the resulting orbits has also been recorded. 


\begin{tabular}{|c|c|}
\hline$I_{0}(\mathrm{rad})$ & 1.1 \\
\hline$E_{0}$ & 0.5 \\
\hline$g_{0}$ & $\frac{\pi}{2}$ \\
\hline$h_{0}$ & $\pi$ \\
\hline$f_{0}$ & $\pi$ \\
\hline$G_{0}$ & 315633 \\
\hline$L_{0}$ & 364462 \\
\hline$H_{0}$ & 143170 \\
\hline \hline$a_{0}(\mathrm{~km})$ & 297.493 \\
\hline
\end{tabular}

Table 2: 433-Eros: initial conditions for frozen orbits

The initial conditions found with this method are transformed back by canonic transformations inverse to the relegating and normalizing transformations of coordinates found in the paper, leading to approximated initial conditions for frozen orbits in the full model. The integration of such system shows a good agreement of the dynamics between the approximated and the full system, namely the resulting orbits for the full system result to be good approximations of frozen orbits. The resulting orbit for 433Eros, for the example in Table (2), is shown below, in the cartesian inertial frame of reference centered in the center of mass of the inhomogeneous body (unit of measure $\mathrm{km}$ ). The order of magnitude of the oscillation of inclination and eccentricity around their initial value is $\Delta$ eccentricity $\sim O\left(10^{-6}\right)$, $\Delta$ eccentricity $\sim O\left(10^{-2}\right)$ deg for at least 20 years. 


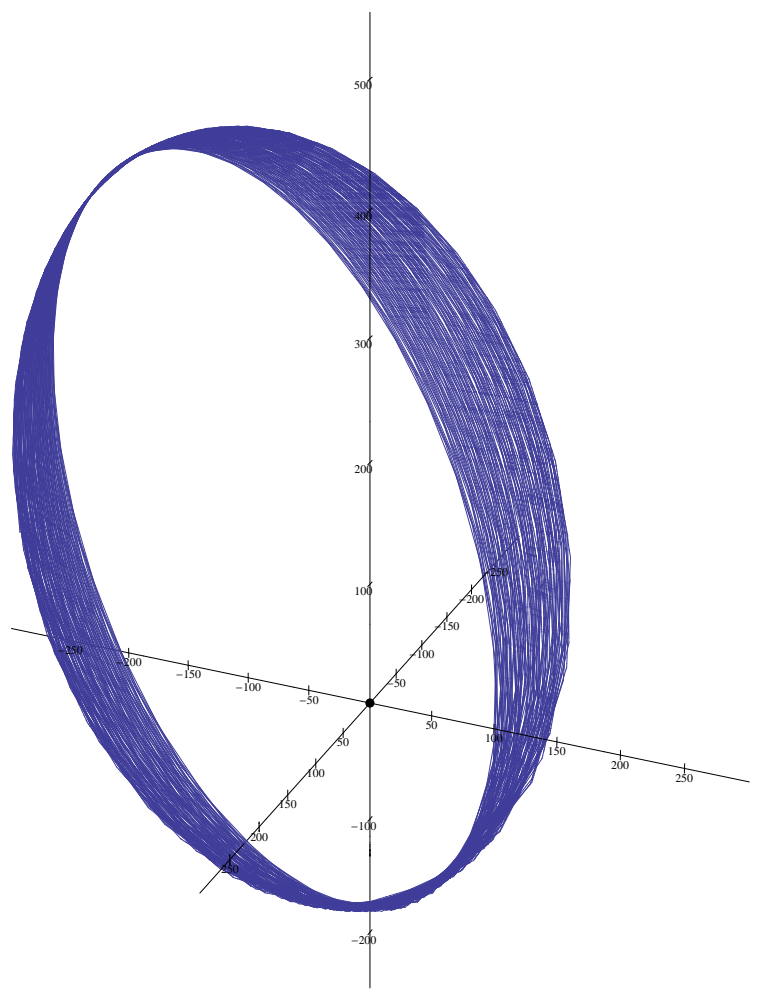

Figure 1: The resulting frozen orbit for $E_{0}=0.5, I_{0}=1.1$ and $g_{0}=-\frac{\pi}{2}$ for 5 years 


\section{Acknowledgements}

A preliminary study on this topic has been developed in collaboration with the Advanced Concepts Team (ACT) at ESTEC funded from the European Space Agency (ESA) as an Ariadna Study Project.

I would like to thank the reviewers for their constructive comments which has led to a substantial improvement in the content of this paper.

\section{References}

Abad, A., Elipe A., Tresaco E.:'Analytical Model to Find Frozen Orbits for a Lunar Orbiter', Journal of Guidance, Control and Dynamics, 32, 888-898, (2009)

Abramowitz, M. and Stegun.,I. A.:' Handbook of Mathematical Functions with Formulas, Graps, and Mathematical Tables', National Bureau Of Standards, Washington (1965)

Arnol'd, Vladimir I.; Kozlov, Valery V.; Neishtadt, Anatoly.:' I. Mathematical aspects of clas- sical and celestial mechanics. Dynamical systems. III.' Encyclopaedia of Mathematical Sciences,Springer-Verlag, Berlin ISBN: 978-3-540-28246-4; 3-540-28246-7(2006)

Brouwer, D.:' Solution of the Problem of the Artificial Satellite Without Drag' Astronomical Journal, 64 (1959)

Carnelli, Gálvez:'ESA's Don Quijote Mission: an Opportunity for the Investigation of an Artificial Impact Crater on an Asteroid, in Proceedings 
of the 25th International Symposium on Space Technology and Science, Kanazawa, Japan, (2006) Paper ISTS 2006-k-26.

Celletti, A.; Chierchia, L.:' KAM stability and celestial mechanics.' Mem. Amer. Math. Soc. 187 , no. 878(2007)

Deprit, A.:'Canonical transformations depending on a small parameter', Celest. Mech. Dyn. Astr., Vol. 1, pp. 12-30 (1969)

Deprit, A., Henrard, J., Price, J. F., Rom, A.:'Birkhoff's Normalization', Being Scientific Research Labs Seattle Wash Mathematics Research Lab. (1969)

Deprit, A.:'The main problem of artificial satellite theory for small and moderate eccentricities', Celestial Mechanics and Dynamical Astronomy, 2, 166-206, (1970)

Deprit, A.:'Delaunay Normalizations', Celestial Mechanics and Dynamical Astronomy, 26, 9-21, (1982)

Deprit, A., Palacián, J., Deprit, E.:'The Relegation Algorithm', Celest. Mech. Dyn. Astr., Vol. 79, pp. 157-182 (2001)

Fahnestock, E., Scheeres, D.,:'Dynamical Characterization and Stabilization of Large Gravity-Tractor Designs', Journal of Guidance, Control, and Dynamics, 31, 501-521, (2008)

Goldstein, Poole, Safko:'Classical Mechanics', Addison Wesley; 3 edition ISBN-13: 978-020165702(2001) 
Hofmann-Wellenhof, B., Moritz, H.:'Physical Geodesy', Springer Wien NewYork (1967)

Lara, M., Scheeres : Stability bounds for three-dimensional motion close to asteroids', AAS/AIAA Space Flight Mechanics Meeting (2002)

Miller, J. K., Konopliv, A. S., Antreasian,P. G., Bordi, J. J. , Chesley, S., Helfrich, C. E., Owen, W. M., Wang, T. C., Williams, B. G., and YeomansD. K. :'Determination of Shape, Gravity, and Rotational State of Asteroid 433 Eros' Jet Propulsion Laboratory, California Institute of Technology, 4800 Oak Grove Drive, Pasadena, California (2001)

Palacián, J.: 1992, Teoría del Satélite Artificial: Armónicos Teserales y su Rele- gación Mediante Simplificaciones Algebraicas," Doctoral Dissertation Universidad de Zaragoza, Spain (1992)

Palacián, J.:'Closed Form normalization of perturbed two-body problems', Chaos, Solitons and Fractals, vol 13, No. 4, pp. 853-87, (2002)

Palacián, J.:'Dynamics of a satellite orbiting a planet with an inhomogeneous gravitational field' Celestial Mech Dyn Astr (2007) 98:219249 DOI $10.1007 / \mathrm{s} 10569-007-9078-5$

Segerman, A. M., Coffey, S.L., :'An analytical theory for tesseral gravitational harmonics., Celestial Mechanics and Dynamical Astronomy, vol. 76, 139-156, (2000)

San-Juan, J.F., Abad, A., Scheeres, D.J., Lara, M.:'A First Order Analytical Solution for Spacecraft Motion about (433) Eros', AIAA, Astrodynamics Specialist Conference, California (2002) 
477 San-Jaun J. F., Abad, A., Lara, M., Scheeres, D. J.,:'First-order analytic 478 solution for Spacecraft about (433) EROS.', Journal of Guidance, Control 479 and Dynamics, vol. 27, No. 2, pp. 290-293, (2004)

480

481

482

483

484

485

486

487

488

Scheeres, D. J. Marzari, F. Tomasella, L. Vanzani, V.,:'ROSETTA mission: satellite orbits around a cometary nucleus', Planetary and Space Science, 46, 649-671, (1998)

Stiefel, E. L.; Scheifele, G.:'Linear and regular celestial mechanics. Perturbed two-body motion, numerical methods, canonical theory.', Die Grundlehren der mathematischen Wissenschaften, Band 174. Springer-Verlag, New York-Heidelberg,(1971)

Whittaker, E.T.:" A treatise on the analytical dynamics of particles and rigid bodies; with an introduction to the problem of three bodies', Cambrige University Press (1917)

Wigner, E.D.:'Group Theory and it's application to the Quantum Mechanics of Atomic Spectra', New York, Academic Press (1959) 


\section{${ }_{492}$ Appendix A: 433-Eros spherical harmonics}

493 The un-normalized spherical harmonic coefficients of 433-Eros are here listed.

${ }_{494}$ This coefficients are the harmonic coefficients gravity solution NEAR15A, a 495 15th degree and order model obtained from radiometric tracking (Doppler 496 and range data) and landmark tracking of the NEAR spacecraft in orbit 497 about Eros. The gravity model includes data from the entire mission begin498 ning with orbit insertion on Feb. 14, 2000 and ending with the first descent 499 maneuver for landing on Feb. 12, 2001 


\begin{tabular}{|c|c|}
\hline$C_{0,0}$ & 1 \\
\hline$C_{1,0}$ & 0 \\
\hline$C_{1,1}$ & 0 \\
\hline$C_{2,0}$ & $-1.65899 \times 10^{-1}$ \\
\hline$C_{2,1}$ & $-2.11454 \times 10^{-6}$ \\
\hline$C_{2,2}$ & $5.31886 \times 10^{-2}$ \\
\hline$C_{3,0}$ & $-5.29244 \times 10^{-3}$ \\
\hline$C_{3,1}$ & $4.38548 \times 10^{-3}$ \\
\hline$C_{3,2}$ & $6.0659 \times 10^{-4}$ \\
\hline$C_{3,3}$ & $-1.4525 \times 10^{-3}$ \\
\hline$C_{4,0}$ & $5.48636 \times 10^{-2}$ \\
\hline$C_{4,1}$ & $-9.52013 \times 10^{-5}$ \\
\hline$C_{4,2}$ & $-3.90614 \times 10^{-3}$ \\
\hline$C_{4,3}$ & $-1.79405 \times 10^{-5}$ \\
\hline$C_{4,4}$ & $3.68808 \times 10^{-4}$ \\
\hline$C_{5,0}$ & $3.09067 \times 10^{-3}$ \\
\hline$C_{5,1}$ & $-2.36787 \times 10^{-3}$ \\
\hline$C_{5,2}$ & $-1.26781 \times 10^{-4}$ \\
\hline$C_{5,3}$ & $1.51169 \times 10^{-4}$ \\
\hline$C_{5,4}$ & $3.86908 \times 10^{-6}$ \\
\hline$C_{5,5}$ & $-2.51307 \times 10^{-5}$ \\
\hline$C_{6,0}$ & $-2.53848 \times 10^{-2}$ \\
\hline$C_{6,1}$ & $-1.91651 \times 10^{-5}$ \\
\hline$C_{6,2}$ & $8.13891 \times 10^{-4}$ \\
\hline$C_{6,3}$ & $5.9664 \times 10^{-6}$ \\
\hline$C_{6,4}$ & $-2.13764 \times 10^{-5}$ \\
\hline$C_{6,5}$ & $-3.93777 \times 10^{-7}$ \\
\hline$C_{6,6}$ & $1.18484 \times 10^{-6}$ \\
\hline
\end{tabular}

\begin{tabular}{|l|l|}
\hline$C_{7,0}$ & $-2.50016 \times 10^{-3}$ \\
\hline$C_{7,1}$ & $1.26047 \times 10^{-3}$ \\
\hline$C_{7,2}$ & $3.82038 \times 10^{-5}$ \\
\hline$C_{7,3}$ & $-3.48143 \times 10^{-5}$ \\
\hline$C_{7,4}$ & $-5.15671 \times 10^{-7}$ \\
\hline$C_{7,5}$ & $1.33563 \times 10^{-6}$ \\
\hline$C_{7,6}$ & $2.25518 \times 10^{-9}$ \\
\hline$C_{7,7}$ & $-1.25528 \times 10^{-7}$ \\
\hline$C_{8,0}$ & $1.53478 \times 10^{-2}$ \\
\hline$C_{8,1}$ & $-3.43765 \times 10^{-5}$ \\
\hline$C_{8,2}$ & $-2.57667 \times 10^{-4}$ \\
\hline$C_{8,3}$ & $-3.12096 \times 10^{-6}$ \\
\hline$C_{8,4}$ & $3.61153 \times 10^{-6}$ \\
\hline$C_{8,5}$ & $8.73471 \times 10^{-8}$ \\
\hline$C_{8,6}$ & $-7.09764 \times 10^{-8}$ \\
\hline$C_{8,7}$ & $-9.71194 \times 10^{-10}$ \\
\hline$C_{8,8}$ & $2.89016 \times 10^{-9}$ \\
\hline$C_{9,0}$ & $1.12427 \times 10^{-3}$ \\
\hline$C_{9,1}$ & $-4.97634 \times 10^{-4}$ \\
\hline$C_{9,2}$ & $-2.57824 \times 10^{-5}$ \\
\hline$C_{9,3}$ & $1.07011 \times 10^{-5}$ \\
\hline$C_{9,4}$ & $-4.14388 \times 10^{-7}$ \\
\hline$C_{9,5}$ & $-1.7556 \times 10^{-7}$ \\
\hline$C_{9,6}$ & $-3.11553 \times 10^{-9}$ \\
\hline$C_{9,7}$ & $5.83725 \times 10^{-9}$ \\
\hline$C_{9,8}$ & $1.43792 \times 10^{-10}$ \\
\hline$C_{9,9}$ & $-2.52185 \times 10^{-10}$ \\
\hline$C_{10,0}$ & $-2.23924 \times 10^{-3}$ \\
\hline
\end{tabular}

\begin{tabular}{|l|c|}
\hline$C_{10,1}$ & $-3.65977 \times 10^{-4}$ \\
\hline$C_{10,2}$ & $8.59725 \times 10^{-5}$ \\
\hline$C_{10,3}$ & $2.44668 \times 10^{-6}$ \\
\hline$C_{10,4}$ & $-2.12904 \times 10^{-8}$ \\
\hline$C_{10,5}$ & $-3.91544 \times 10^{-8}$ \\
\hline$C_{10,6}$ & $1.06018 \times 10^{-8}$ \\
\hline$C_{10,7}$ & $6.6781 \times 10^{-10}$ \\
\hline$C_{10,8}$ & $-1.03388 \times 10^{-10}$ \\
\hline$C_{10,9}$ & $-2.93031 \times 10^{-11}$ \\
\hline$C_{10,10}$ & $4.93363 \times 10^{-12}$ \\
\hline$C_{11,0}$ & $1.04666 \times 10^{-2}$ \\
\hline$C_{11,1}$ & $3.72982 \times 10^{-4}$ \\
\hline$C_{11,2}$ & $3.37686 \times 10^{-6}$ \\
\hline$C_{11,3}$ & $1.80367 \times 10^{-6}$ \\
\hline$C_{11,4}$ & $-5.5386 \times 10^{-7}$ \\
\hline$C_{11,5}$ & $7.57115 \times 10^{-8}$ \\
\hline$C_{11,6}$ & $2.19576 \times 10^{-9}$ \\
\hline$C_{11,7}$ & $5.19815 \times 10^{-11}$ \\
\hline$C_{11,8}$ & $3.75133 \times 10^{-11}$ \\
\hline$C_{11,9}$ & $1.74028 \times 10^{-11}$ \\
\hline$C_{11,10}$ & $-2.76742 \times 10^{-13}$ \\
\hline$C_{11,11}$ & $-2.57971 \times 10^{-13}$ \\
\hline$C_{12,0}$ & $1.71922 \times 10^{-3}$ \\
\hline$C_{12,1}$ & $3.7954 \times 10^{-4}$ \\
\hline$C_{12,2}$ & $1.55553 \times 10^{-4}$ \\
\hline$C_{12,3}$ & $6.86842 \times 10^{-6}$ \\
\hline$C_{12,4}$ & $2.99064 \times 10^{-7}$ \\
\hline$C_{12,5}$ & $-8.38626 \times 10^{-8}$ \\
\hline & \\
\hline
\end{tabular}




\begin{tabular}{|l|c|}
\hline$C_{12,6}$ & $4.07023 \times 10^{-9}$ \\
\hline$C_{12,7}$ & $-1.60746 \times 10^{-10}$ \\
\hline$C_{12,8}$ & $1.86662 \times 10^{-11}$ \\
\hline$C_{12,9}$ & $-4.62122 \times 10^{-12}$ \\
\hline$C_{12,10}$ & $-5.72445 \times 10^{-13}$ \\
\hline$C_{12,11}$ & $2.02689 \times 10^{-14}$ \\
\hline$C_{12,12}$ & $8.12551 \times 10^{-15}$ \\
\hline$C_{13,0}$ & $2.75545 \times 10^{-2}$ \\
\hline$C_{13,1}$ & $-2.9199 \times 10^{-3}$ \\
\hline$C_{13,2}$ & $-2.02593 \times 10^{-6}$ \\
\hline$C_{13,3}$ & $6.84023 \times 10^{-6}$ \\
\hline$C_{13,4}$ & $3.23691 \times 10^{-7}$ \\
\hline$C_{13,5}$ & $-3.54904 \times 10^{-8}$ \\
\hline$C_{13,6}$ & $2.59498 \times 10^{-10}$ \\
\hline$C_{13,7}$ & $4.03437 \times 10^{-11}$ \\
\hline$C_{13,8}$ & $-1.37277 \times 10^{-11}$ \\
\hline$C_{13,9}$ & $-7.31327 \times 10^{-13}$ \\
\hline$C_{13,10}$ & $-7.27471 \times 10^{-14}$ \\
\hline$C_{13,11}$ & $2.30772 \times 10^{-14}$ \\
\hline$C_{13,12}$ & $2.58196 \times 10^{-16}$ \\
\hline$C_{13,13}$ & $-2.18667 \times 10^{-16}$ \\
\hline$C_{14,0}$ & $-1.53377 \times 10^{-2}$ \\
\hline$C_{14,1}$ & $7.66068 \times 10^{-4}$ \\
\hline$C_{14,2}$ & $2.96292 \times 10^{-4}$ \\
\hline$C_{14,3}$ & $5.32869 \times 10^{-6}$ \\
\hline$C_{14,4}$ & $-5.87731 \times 10^{-7}$ \\
\hline$C_{14,5}$ & $-5.31799 \times 10^{-8}$ \\
\hline$C_{14,6}$ & $2.30096 \times 10^{-9}$ \\
\hline
\end{tabular}

\begin{tabular}{|l|c|}
\hline$C_{14,7}$ & $-7.86604 \times 10^{-11}$ \\
\hline$C_{14,8}$ & $-7.59718 \times 10^{-12}$ \\
\hline$C_{14,9}$ & $-1.0193 \times 10^{-13}$ \\
\hline$C_{14,10}$ & $5.29761 \times 10^{-14}$ \\
\hline$C_{14,11}$ & $7.4175 \times 10^{-15}$ \\
\hline$C_{14,12}$ & $-9.24318 \times 10^{-16}$ \\
\hline$C_{14,13}$ & $-2.2948 \times 10^{-17}$ \\
\hline$C_{14,14}$ & $1.81628 \times 10^{-17}$ \\
\hline$C_{15,0}$ & $2.06404 \times 10^{-2}$ \\
\hline$C_{15,1}$ & $-2.65164 \times 10^{-3}$ \\
\hline$C_{15,2}$ & $9.46812 \times 10^{-6}$ \\
\hline$C_{15,3}$ & $-3.69445 \times 10^{-6}$ \\
\hline$C_{15,4}$ & $3.18757 \times 10^{-7}$ \\
\hline$C_{15,5}$ & $-2.84101 \times 10^{-8}$ \\
\hline$C_{15,6}$ & $-1.9038 \times 10^{-10}$ \\
\hline$C_{15,7}$ & $-6.24463 \times 10^{-11}$ \\
\hline$C_{15,8}$ & $-1.06965 \times 10^{-11}$ \\
\hline$C_{15,9}$ & $-2.61478 \times 10^{-13}$ \\
\hline$C_{15,10}$ & $5.55852 \times 10^{-16}$ \\
\hline$C_{15,11}$ & $-1.64954 \times 10^{-15}$ \\
\hline$C_{15,12}$ & $4.81127 \times 10^{-17}$ \\
\hline$C_{15,13}$ & $2.5553 \times 10^{-17}$ \\
\hline$C_{15,14}$ & $5.60796 \times 10^{-19}$ \\
\hline$C_{15,15}$ & $-5.49434 \times 10^{-19}$ \\
\hline
\end{tabular}




\begin{tabular}{|c|c|}
\hline$S_{0,0}$ & 0 \\
\hline$S_{1,0}$ & 0 \\
\hline$S_{1,1}$ & 0 \\
\hline$S_{2,0}$ & 0 \\
\hline$S_{2,1}$ & $-1.80744 \times 10^{-7}$ \\
\hline$S_{2,2}$ & $-1.81446 \times 10^{-2}$ \\
\hline$S_{3,0}$ & 0 \\
\hline$S_{3,1}$ & $3.63836 \times 10^{-3}$ \\
\hline$S_{3,2}$ & $-2.40395 \times 10^{-4}$ \\
\hline$S_{3,3}$ & $-1.68328 \times 10^{-3}$ \\
\hline$S_{4,0}$ & 0 \\
\hline$S_{4,1}$ & $1.29913 \times 10^{-4}$ \\
\hline$S_{4,2}$ & $1.0351 \times 10^{-3}$ \\
\hline$S_{4,3}$ & $-7.12399 \times 10^{-6}$ \\
\hline$S_{4,4}$ & $-1.92384 \times 10^{-4}$ \\
\hline$S_{5,0}$ & 0 \\
\hline$S_{5,1}$ & $-1.04273 \times 10^{-3}$ \\
\hline$S_{5,2}$ & $6.17062 \times 10^{-5}$ \\
\hline$S_{5,3}$ & $1.16925 \times 10^{-4}$ \\
\hline$S_{5,4}$ & $-5.43531 \times 10^{-6}$ \\
\hline$S_{5,5}$ & $-1.43782 \times 10^{-5}$ \\
\hline$S_{6,0}$ & 0 \\
\hline$S_{6,1}$ & $-9.74106 \times 10^{-5}$ \\
\hline$S_{6,2}$ & $-1.48126 \times 10^{-4}$ \\
\hline$S_{6,3}$ & $1.56395 \times 10^{-6}$ \\
\hline$S_{6,4}$ & $1.56395 \times 10^{-6}$ \\
\hline$S_{6,5}$ & $3.86799 \times 10^{-8}$ \\
\hline$S_{6,6}$ & $-3.73278 \times 10^{-7}$ \\
\hline
\end{tabular}

\begin{tabular}{|c|c|}
\hline$S_{7,0}$ & 0 \\
\hline$S_{7,1}$ & $5.15445 \times 10^{-4}$ \\
\hline$S_{7,2}$ & $-1.97429 \times 10^{-5}$ \\
\hline$S_{7,3}$ & $-2.02322 \times 10^{-5}$ \\
\hline$S_{7,4}$ & $6.94006 \times 10^{-7}$ \\
\hline$S_{7,5}$ & $6.72634 \times 10^{-7}$ \\
\hline$S_{7,6}$ & $-3.44172 \times 10^{-8}$ \\
\hline$S_{7,7}$ & $-4.07766 \times 10^{-8}$ \\
\hline$S_{8,0}$ & 0 \\
\hline$S_{8,1}$ & $-1.24043 \times 10^{-5}$ \\
\hline$S_{8,2}$ & $2.30047 \times 10^{-6}$ \\
\hline$S_{8,3}$ & $-3.22691 \times 10^{-7}$ \\
\hline$S_{8,4}$ & $-6.27617 \times 10^{-7}$ \\
\hline$S_{8,5}$ & $-1.85513 \times 10^{-8}$ \\
\hline$S_{8,6}$ & $6.66802 \times 10^{-10}$ \\
\hline$S_{8,7}$ & $-3.57144 \times 10^{-10}$ \\
\hline$S_{8,8}$ & $1.74786 \times 10^{-9}$ \\
\hline$S_{9,0}$ & 0 \\
\hline$S_{9,1}$ & $-8.17618 \times 10^{-5}$ \\
\hline$S_{9,2}$ & $-1.31237 \times 10^{-5}$ \\
\hline$S_{9,3}$ & $7.54724 \times 10^{-6}$ \\
\hline$S_{9,4}$ & $-2.35188 \times 10^{-7}$ \\
\hline$S_{9,5}$ & $-1.00222 \times 10^{-7}$ \\
\hline$S_{9,6}$ & $1.12056 \times 10^{-9}$ \\
\hline$S_{9,7}$ & $1.6534 \times 10^{-9}$ \\
\hline$S_{9,8}$ & $-2.32921 \times 10^{-11}$ \\
\hline$S_{9,9}$ & $-5.56697 \times 10^{-11}$ \\
\hline$S_{10,0}$ & 0 \\
\hline & \\
\hline
\end{tabular}

\begin{tabular}{|l|l|}
\hline$S_{10,1}$ & $6.94286 \times 10^{-4}$ \\
\hline$S_{10,2}$ & $-4.56443 \times 10^{-5}$ \\
\hline$S_{10,3}$ & $2.62557 \times 10^{-6}$ \\
\hline$S_{10,4}$ & $-4.14985 \times 10^{-7}$ \\
\hline$S_{10,5}$ & $-5.74199 \times 10^{-8}$ \\
\hline$S_{10,6}$ & $6.45742 \times 10^{-9}$ \\
\hline$S_{10,7}$ & $-7.47668 \times 10^{-10}$ \\
\hline$S_{10,8}$ & $-4.99191 \times 10^{-12}$ \\
\hline$S_{10,9}$ & $9.74982 \times 10^{-13}$ \\
\hline$S_{10,10}$ & $5.59573 \times 10^{-12}$ \\
\hline$S_{11,0}$ & 0 \\
\hline$S_{11,1}$ & $-8.17892 \times 10^{-4}$ \\
\hline$S_{11,2}$ & $-6.92074 \times 10^{-5}$ \\
\hline$S_{11,3}$ & $-1.13881 \times 10^{-6}$ \\
\hline$S_{11,4}$ & $-4.84678 \times 10^{-7}$ \\
\hline$S_{11,5}$ & $8.37324 \times 10^{-8}$ \\
\hline$S_{11,6}$ & $-1.09462 \times 10^{-9}$ \\
\hline$S_{11,7}$ & $-2.46115 \times 10^{-10}$ \\
\hline$S_{11,8}$ & $-2.79264 \times 10^{-11}$ \\
\hline$S_{11,9}$ & $9.02775 \times 10^{-12}$ \\
\hline$S_{11,10}$ & $1.31812 \times 10^{-13}$ \\
\hline$S_{11,11}$ & $-1.94565 \times 10^{-13}$ \\
\hline$S_{12,0}$ & 0 \\
\hline$S_{12,1}$ & $1.50676 \times 10^{-3}$ \\
\hline$S_{12,2}$ & $9.64141 \times 10^{-5}$ \\
\hline$S_{12,3}$ & $2.73675 \times 10^{-6}$ \\
\hline$S_{12,4}$ & $-2.39721 \times 10^{-7}$ \\
\hline$S_{12,5}$ & $-3.08972 \times 10^{-8}$ \\
\hline
\end{tabular}




\begin{tabular}{|c|c|}
\hline$S_{12,6}$ & $9.18684 \times 10^{-9}$ \\
\hline$S_{12,7}$ & $-5.56246 \times 10^{-10}$ \\
\hline$S_{12,8}$ & $5.98262 \times 10^{-12}$ \\
\hline$S_{12,9}$ & $1.23035 \times 10^{-13}$ \\
\hline$S_{12,10}$ & $-7.24925 \times 10^{-13}$ \\
\hline$S_{12,11}$ & $1.701 \times 10^{-14}$ \\
\hline$S_{12,12}$ & $1.63895 \times 10^{-14}$ \\
\hline$S_{13,0}$ & 0 \\
\hline$S_{13,1}$ & $-1.24564 \times 10^{-3}$ \\
\hline$S_{13,2}$ & $1.54632 \times 10^{-5}$ \\
\hline$S_{13,3}$ & $-6.74004 \times 10^{-7}$ \\
\hline$S_{13,4}$ & $-1.19607 \times 10^{-6}$ \\
\hline$S_{13,5}$ & $6.26074 \times 10^{-9}$ \\
\hline$S_{13,6}$ & $-1.26688 \times 10^{-10}$ \\
\hline$S_{13,7}$ & $-7.5178 \times 10^{-13}$ \\
\hline$S_{13,8}$ & $-1.60844 \times 10^{-11}$ \\
\hline$S_{13,9}$ & $-9.10394 \times 10^{-14}$ \\
\hline$S_{13,10}$ & $-7.19669 \times 10^{-15}$ \\
\hline$S_{13,11}$ & $-5.20369 \times 10^{-15}$ \\
\hline$S_{13,12}$ & $-1.01803 \times 10^{-16}$ \\
\hline$S_{13,13}$ & $-4.21829 \times 10^{-16}$ \\
\hline$S_{14,0}$ & 0 \\
\hline$S_{14,1}$ & $8.65044 \times 10^{-4}$ \\
\hline$S_{14,2}$ & $1.51562 \times 10^{-4}$ \\
\hline$S_{14,3}$ & $4.31479 \times 10^{-7}$ \\
\hline$S_{14,4}$ & $1.77234 \times 10^{-7}$ \\
\hline$S_{14,5}$ & $-1.76094 \times 10^{-9}$ \\
\hline$S_{14,6}$ & $4.30073 \times 10^{-9}$ \\
\hline
\end{tabular}

\begin{tabular}{|l|l|}
\hline$S_{14,7}$ & $-2.43475 \times 10^{-10}$ \\
\hline$S_{14,8}$ & $-1.42072 \times 10^{-11}$ \\
\hline$S_{14,9}$ & $4.1348 \times 10^{-13}$ \\
\hline$S_{14,10}$ & $8.33334 \times 10^{-15}$ \\
\hline$S_{14,11}$ & $6.89565 \times 10^{-16}$ \\
\hline$S_{14,12}$ & $-3.88959 \times 10^{-16}$ \\
\hline$S_{14,13}$ & $3.71979 \times 10^{-18}$ \\
\hline$S_{14,14}$ & $2.08219 \times 10^{-17}$ \\
\hline$S_{15,0}$ & 0 \\
\hline$S_{15,1}$ & $-6.5828 \times 10^{-5}$ \\
\hline$S_{15,2}$ & $9.63909 \times 10^{-5}$ \\
\hline$S_{15,3}$ & $9.90187 \times 10^{-7}$ \\
\hline$S_{15,4}$ & $-7.56365 \times 10^{-7}$ \\
\hline$S_{15,5}$ & $-3.05489 \times 10^{-8}$ \\
\hline$S_{15,6}$ & $-2.45565 \times 10^{-10}$ \\
\hline$S_{15,7}$ & $-1.12172 \times 10^{-11}$ \\
\hline$S_{15,8}$ & $2.66204 \times 10^{-12}$ \\
\hline$S_{15,9}$ & $-2.21231 \times 10^{-14}$ \\
\hline$S_{15,10}$ & $-7.67107 \times 10^{-15}$ \\
\hline$S_{15,11}$ & $-2.08224 \times 10^{-15}$ \\
\hline$S_{15,12}$ & $4.21957 \times 10^{-17}$ \\
\hline$S_{15,13}$ & $1.21087 \times 10^{-17}$ \\
\hline$S_{15,14}$ & $-3.91552 \times 10^{-19}$ \\
\hline$S_{15,15}$ & $-4.94421 \times 10^{-19}$ \\
\hline
\end{tabular}

